\title{
La recepción argentina de la Phänomenologie des Geistes en la década de 1960
}

\author{
The Argentine Reception of the Phänomenologie des Geistes \\ in the 1960s
}

Milton Abellón

Resumen: El presente trabajo aborda de manera exploratoria la recepción argentina de la Pbänomenologie des Geistes de 1807 (Phä.) en los años 60. Nuestro propósito consiste en examinar las obras más destacadas, delimitar los lineamientos interpretativos, las problemáticas de interés más acuciantes, los interlocutores principales y las obras disponibles. En primer lugar, presentamos el tema y señalamos los antecedentes más relevantes. En segundo lugar, nos detenemos en la década de 1960. Sostenemos que los intereses fundamentales de esta etapa se dividen en cuatro temáticas: $i$. la dialéctica de las figuras de la conciencia, ii. los escritos juveniles de Hegel y su relación con la Phä., iii. las interpretaciones francesas y $i v$ la relación entre Hegel y Marx. Examinamos las obras más significativas de este periodo a la luz de esta estructuración temática. Finalmente, en tercer lugar, realizamos algunas consideraciones conclusivas que nos permiten dar con una caracterización general de la cuestión desarrollada.

Palabras clave: Fenomenología del espiritu, Argentina, Recepción, Década de 1960.

Abstract: This paper studies in an exploratory way the argentine reception of the Phänomenologie des Geistes of 1807 (Phä.) in the 1960s. Our purpose is to examine the most important works, to delimit the interpretative guidelines readings, the most urgent problems, the issues of interest, the main interlocutors, and the available works. Firstly, we introduce the subject and point out the most relevant antecedents. Secondly, we focus on the 1960s. We argue that the fundamental interests of this period are divided into four themes: $i$. the dialectics of the figures

\footnotetext{
* Doctor en Filosofía por la Universidad de Buenos Aires (UBA) e Investigador del Consejo Nacional de Investigaciones Científicas y Técnicas (CONICET) y del Instituto Ezequiel de Olaso del Centro de Investigaciones Filosóficas (CIF). Se desempeña como Docente del Departamento de Filosofía de la Facultad de Filosofía y Letras de la UBA. Su área de investigación son los estudios hegelianos, especialmente las recepciones contemporáneas de Hegel. Dirección electrónica: milton.abellon@gmail.com
} 
of consciousness, ii. Hegel's youthful writings and their relation to the Phä., iii. the French interpretations, and $i v$. the relation between Hegel and Marx. We examine the most significant works of this period in the light of this thematic structure. Finally, in the third place, we make some concluding considerations that allow us to give a general characterization of the subject developed.

Keywords: Phenomenology of Spirit, Argentine, Reception, 1960s.

\section{Presentación y antecedentes fundamentales}

El presente trabajo aborda de manera exploratoria la recepción argentina de la Phänomenologie des Geistes de 1807 (Phä.) en los años 60, momento en que se publica una variedad de textos fuente de distintos autores argentinos, lo que brinda la amplitud bibliográfica para realizar el tipo de aproximación que nos proponemos. Nuestro interés se centra en delimitar los lineamientos interpretativos en juego, las problemáticas de interés más acuciantes, los interlocutores principales y las obras disponibles.

El tercer periodo (1950-1982/1983) de la recepción de Hegel en nuestro país, en el que se enmarcan los años que estudiamos, está inmediatamente antecedido por el Primer Congreso Nacional de Filosofía celebrado en 1949 en Mendoza en el marco de la presidencia de Juan Domingo Perón. ${ }^{2}$ Con este evento, que da término al segundo periodo (1918-1949), culmina una etapa importante de la institucionalización de la filosofía argentina, en la tuvieron lugar las primeras investigaciones con estándares académicos y cuyos intereses principales fueron signados por la fenomenología y el existencialismo, por un lado, y por la renovación de los estudios tomistas y hegelianos, por otro. Es en este segundo periodo en el que se establece y luego se consolida la transición de varios intelectuales al hegelianismo, como son los casos de Nimio de Anquín y Juan Ramón Sepich, ambos provenientes del tomismo. ${ }^{3}$

\footnotetext{
2 Para la periodización de la recepción argentina de Hegel véase el texto de Eduardo Assalone incluido en el presente Dossier: "Hegel en Argentina. Un estado de la cuestión y una propuesta de periodización".

3 Podetti, Ramiro, "Advertencia preliminar", en Amalia, Podetti, Comentario a la Introducción a la Fenomenología del Espíritu, Buenos Aires, Biblos, 2007, p. 28 [1534]. Véase también Alberini, Coriolano, "Prólogo", en Luis, Farré, Cincuenta años de filosofía en Argentina, Buenos Aires, Peuser, 1958, pp. 7-19.
} 
En este contexto de transición a un nuevo periodo, la Phä. toma un lugar destacado en los estudios hegelianos argentinos. Miguel Ángel Virasoro ${ }^{4}$, en sus palabras esgrimidas en homenaje a Guido de Ruggiero (discípulo del neohegeliano Giovanni Gentile) en el Primer Congreso, expresa que el estado actual de la filosofía está marcado por un "interés creciente" ${ }^{5}$ por la $P h \ddot{a}$, así como por su recomprensión y renovación, tal como lo ponen de manifiesto no sólo las clásicas figuras de Koyré, Kojève e Hyppolite, sino también otros intelectuales de gran talla como Eugen Fink, Ludwig Landgrebe y Enrico de Negri. Según el autor argentino, que en 1946 publica la primera traducción castellana de L'être et le néant, lo que muestra la revaloración de la Phä. es la compatibilidad de la filosofía de Hegel con la fenomenología, la filosofía de la existencia y el existencialismo, cuyos máximos exponentes eran Husserl, Heidegger y Sartre.

En 1949 sucede otro acontecimiento importante, pues se publica en el número 3 de la Revista del Instituto de Filosofía de la Facultad de Filosofía y Letras de la Universidad de Buenos Aires, Serie de Textos Filosóficos. Filosofía Moderna, la que sería la primera traducción argentina de la Phä. - de autoría desconocida. ${ }^{6}$ Se trata de una traducción parcial, pero directa del alemán, de la parte de la sección B del capítulo IV dedicada a la conciencia desdichada (unglückliche Bewnßtsein). La traducción se titula "La conciencia infeliz". La fuente alemana es la edición de Von H. Glockner, Sämtliche Werke. Jubiläumsausgabe in zwanæig Bänden, Bd. II, 1932. La edición argentina toma en consideración además las traducciones disponibles en otros idiomas, específicamente la italiana de Enrico de Negri, cuya primera edición data de 1933 y la segunda de 1949, la francesa de J. Hyppolite,

\footnotetext{
${ }^{4}$ Virasoro (1900-1966) fue un abogado y filósofo que se desempeñó como profesor en la Cátedra de Historia de la Filosofía Moderna y Contemporánea en la Facultad de Filosofía y Letras de la Universidad de Buenos Aires y en la de Filosofía Contemporánea de la Universidad del Litoral. En 1955, en el marco de la Dictadura Militar liderada por Pedro Eugenio Aramburu, quien sucede a Eduardo Lonardi, es expulsado de ambas cátedras, pudiendo retomar sus actividades en la Universidad Nacional del Sur y en la Universidad Nacional de Cuyo en 1960.

5 Virasoro, Miguel Ángel, "Guido de Ruggiero”, en Actas del Primer Congreso Nacional de Filosofía, Tomo I, Universidad Nacional de Cuyo, Mendoza, 1950, pp. 609-610 [607-610].

${ }^{6} \mathrm{La}$ aparición de esta traducción es posterior al Congreso, pues se imprime el 20 de diciembre de 1949, mientras aquel evento tiene lugar del 30 de marzo al 9 de abril. En este trabajo no nos ocupamos de evaluar las traducciones, pues ello requeriría un abordaje de los textos que excede nuestros propósitos ya consignados.
} 
publicada en 1939 y reeditada en 1941, y la inglesa de J. B. Baillie, aparecida en 1910 y reeditada en $1931 .^{7}$

Esta publicación significa un gran aporte no sólo en el campo de las traducciones argentinas, sino también en lo que respecta a las traducciones de la Phä. al idioma español en general. Para 1949 sólo se contaba con la traducción del Prólogo, de la Introducción y del capítulo VIII, "El saber absoluto" realizada por el español Xavier Zubiri y publicada en la Revista de Occidente en $1935 .{ }^{8}$ En este sentido, "La conciencia infeliz" constituye la primera traducción al castellano de esta sección de la Phä., representando una contribución de envergadura a nivel mundial.

Aunque de autoría desconocida, la traducción argentina fue revisada por la Dirección del Instituto de Filosofía, que se encontraba desde 1948 en manos de uno de los intelectuales más destacados, Carlos Astrada (18941970); quien será depuesto de este cargo y de sus funciones docentes en esta institución en 1956, en el marco de la dictadura militar liderada por Pedro Eugenio Aramburu. ${ }^{9}$ La traducción está antecedida también por "Génesis

\footnotetext{
${ }^{7}$ La traducción argentina no especifica qué ediciones consulta. Suponemos que se utilizaron las que por entonces eran más recientes, al menos en lo que hace a la francesa e inglesa, pues la italiana es del mismo año que la traducción castellana.

${ }^{8}$ Esta traducción parcial es directa del alemán y toma como fuente la Sämtliche Werke, Hg. von G. Lasson, später J. Hoffmeister Leipzig, 1921 (1907). La edición española que por entonces oficiaba de fuente para el tema del espíritu era una traducción indirecta de la Filosofía del Espiritu realizada por Eduardo Barrioberro y Herrán (1ra. Edición, Madrid, Daniel Jorro, 1907; 2da. edición, Buenos Aires, Anaconda; 3ra y 4ta. ediciones, Buenos Aires, Claridad, 1969 y 2006) a partir de la edición francesa de Augusto Véra (París, Germer Baillière, 1869 y 1897), la cual está basada en la edición alemana Werke. Vollständige Ausgabe durch einen Verein von Freunden des Verewigten. Bd. II, Berlín, 1832 (2da. Ed. 1841). Juan Ramón Sepich, en el marco de sus cursos de 1971 dictados en la Universidad de Cuyo, realiza un comentario al Prefacio de la $P h \ddot{a}$. que permanecería inédito, siendo publicada una versión de éste al año siguiente como Propedéntica filosófica. Prefacio al "Sistema de la ciencia" de Hegel, Buenos, Aires, Itinerarium.

${ }^{9}$ En su primer año de dirección, Astrada funda la Revista del Instituto, Cuadernos de Filosofía, la cual continúa vigente. La Serie textos Filosóficos publicaba inéditos en lengua castellana. Para 1949, se desempeñaba como profesor de Historia de la Filosofía Moderna, Contemporánea, Metafísica y Gnoseología en la Facultad de Filosofía y Letras de la Universidad de Buenos Aires, aunque también fue profesor de Ética en la Facultad de Humanidades y Ciencias de la Educación de la Universidad Nacional de La Plata de 1936 a 1947 y profesor de Sociología y Lógica en la Universidad Nacional del Sur de 1959 a 1961.
} 
dialéctica de la conciencia infeliz" de Astrada. El pensamiento de este autor, quien de 1927 a 1931 se formó en Köln y Freiburg con Heidegger, Husserl, Scheler y Hartmann, estuvo fuertemente influenciado por la fenomenología, la filosofía existencial y el existencialismo y, a partir de mediados de la década de 1950, por el marxismo.

El texto de 1949 presenta un lineamiento interpretativo de corte existencial que anticipa la perspectiva histórica que prevalecerá en trabajos posteriores. En un gesto que recuerda a Le malheur de la conscience dans la philosophie de Hegel de Jean Wahl, que aquí no menciona, define a la conciencia infeliz como "la figura central de la Fenomenología". ${ }^{10}$ Pues desarrolla la conciencia misma de la escisión entre el hombre y el mundo, y la búsqueda de reposo -sc. de la unidad-, como un movimiento que caracteriza a la totalidad de la experiencia fenomenológica. Sin embargo, siguiendo la lectura que el idealista estadounidense Josiah Royce presenta en Lectures on Modern Idealism ${ }^{11}$, aquello no se reduce a la conciencia individual, sino que compete también a la búsqueda que el espíritu universal realiza de sí a través de sus desgarramientos.

Los años 50, que inician un periodo en el que la institucionalización y la producción filosófica se ve entorpecida por los acontecimientos políticos, están signados por el pensamiento de Astrada. Tras el derrocamiento de Perón a mediados de esta década, mientras en el país se desarrolla la dictadura militar autodenominada Revolución libertadora (1955-1958), Astrada comienza a estar fuertemente influenciado por el marxismo -al igual que otros intelectuales argentinos-, llegando a integrar en 1956 la Delegación del Partido Comunista en el viaje a la URSS. Entre sus obras se destacan, en primer lugar, Hegely la dialéctica, publicada en 1956 por Kairós (Buenos Aires). El libro se centra fundamentalmente en la cuestión de la dialéctica en la lógica de Hegel y establece correlaciones con la Phä. Es inmediatamente posterior al curso de Wissenschaft der Logik que dicta en 1955 en la Facultad de Filosofía y Letras de la Universidad de Buenos Aires, el cual constituyó su último curso en esta institución. En segundo lugar, El marxismo y las escatologías, publicado en 1957 por Procyon

10 Astrada, Carlos, "Génesis dialéctica de la conciencia infeliz", Serie Textos Filosóficos. Filosofía Moderna, 3 (1949), p. 6.

${ }^{11}$ El libro de Josiah Royce se publica en 1919 por Yale University Press y luego se reedita en 1923 y 1934. Astrada sigue la traducción castellana de Vicente Quintero, publicada en 1945 por la editorial Imán (Buenos Aires). 
(Buenos Aires), que patentiza paradigmáticamente su giro al marxismo. ${ }^{12}$ En tercer lugar, ya en el marco de la presidencia de Arturo Frondizi (19581962), se destaca Marx y Hegel. Trabajo y alienación en la "Fenomenología" y en los "Manuscritos", que se publica en 1958 en oportunidad del 150 aniversario de la Phä., contando con una introducción fechada precisamente en 1957.13

Este último texto, el más relevante para nuestra temática, aborda la concepción hegeliana del trabajo a la luz de la valoración crítica de Marx, presentando un lineamiento interpretativo histórico de corte marxista. A partir del rechazo a las lecturas idealistas de la filosofía hegeliana representadas por Croce y Gentile ${ }^{14}$, que priorizan lo lógico e ideal del concepto puro por sobre lo concreto e histórico, Astrada entiende la dialéctica como apertura a la estructura misma de lo real y concreto de la realidad histórica, cuyo origen y génesis se halla en la actividad formativa del trabajo humano y se consolida en la sociedad burguesa. Ahora bien, al respecto, el autor argentino señala tres diferencias fundamentales entre los planteos de Hegel y Marx. La primera es que, mientras Hegel expone el lado positivo del trabajo, su carácter transformador y productivo, junto a su valor antropógeno y liberador, Marx enfatiza el lado negativo, la alienación del obrero y de la especie humana en general. La segunda diferencia es que Marx comprende el trabajo como trabajo humano, lo que hace del hombre real y efectivo el protagonista de la historia. En cambio, Hegel reduce el trabajo humano a mero momento del espíritu absoluto, verdadero protagonista de la historia universal. En este sentido, la concepción hegeliana del trabajo es abstracta e ideal. Finalmente, en tercer lugar, señala que en Hegel la libertad no es efectiva, pues queda limitada al conocimiento de ella, es decir, a un plano cognitivo. Por el contrario, Marx sitúa la liberación y la realización efectiva de la libertad en la praxis revolucionaria, que emancipa al obrero de la coacción y la necesidad externa del trabajo, a

\footnotetext{
12 Seguimos aquí a Ramaglia, Dante, “Apropiación crítica de Hegel en la filosofía argentina contemporánea", Cuyo. Anuario de Filosofía Argentina y Americana, 24 (2007), pp. 128-130.

${ }^{13}$ La edición alemana de la Phä. que utiliza es la misma que en la traducción de 1949. Cf. supra. En el caso de Marx, se refiere a National Oekonomie und Philosophie, Verlag Kiepenheuer, Köln, 1950, texto que reproduce el de la Marx-Engels-Ausgabe. Instituto Marx-Engels-Lenin, Moscú. Bd. 3, 1932.

${ }^{14}$ De Croce, Astrada se refiere específicamente la edición de 1932 de Ciò che è vivo e ciò che è morto della filosofia di Hegel, publicada en Saggio sullo Hegel. De Gentile, a la 2da. edición de La Riforma della Dialettica Hegeliana, publicada en 1923.
} 
la vez que establece una organización social en la que el intercambio de las materias con el mundo natural queda bajo control comunitario.

En lo que sigue, nos ocuparemos de los años 60, en la que la presencia de Astrada sigue vigente, aunque se deja oír una cantidad de voces que consolidan a la Phä. como centro de interés de los estudios hegelianos argentinos.

\section{La Phänomenologie en la década del 60}

La década de 1960 se inicia con la presidencia de Arturo Frondizi, quien es derrocado por el Golpe de Estado de 1962, asumiendo posteriormente el poder José María Guido (1962-1963). Son años convulsionados para el país, en los cuales la producción filosófica sobre la $P h \ddot{a}$. parece interrumpirse, volviendo a retomar vuelo estable a mediados de la década en el marco de la presidencia constitucional de Arturo Umberto Illia (1963-1966), quien gana las elecciones bajo proscripción del peronismo y el comunismo. Nuestro propósito no es hacer un análisis crítico de las publicaciones del periodo, las cuales abordan la Phä. de modo apropiativo. En algunos casos, incluso, se limitan a reponer interpretaciones europeas influyentes. Nuestro objetivo consiste en examinar las obras más destacadas, organizarlas en función de sus intereses más apremiantes, delimitando los problemas de los que se ocupan, sus líneas interpretativas y tesis generales, así como también sus interlocutores principales y las ediciones de las obras que utilizan. Desde este lugar, sostenemos que los intereses fundamentales de las publicaciones más significativas de esta etapa se dividen en cuatro temáticas: $i$. la dialéctica de las figuras de la conciencia, $i i$. los escritos juveniles de Hegel y su relación con la Phä., iii. las interpretaciones francesas y $i v$. la relación entre Hegel y Marx.

Una cuestión preliminar que cabe destacar antes de desarrollar dichos puntos, es que Amalia Podetti ${ }^{15}$ traduce la Introducción a la Phä. en 1966 y realiza un comentario detallado de la misma en 1968. Ambos textos se

\footnotetext{
15 Podetti (1928-1979), discípula de Carlos Astrada y Andrés Mercado Vera, ingresa a la carrera de Filosofía de Facultad de Filosofía y Letras de la Universidad de Buenos Aires en 1948 y se gradúa en 1956 bajo la dirección de Eugenio Pucciarelli. En la década del 60, asiste a diversos cursos de formación en París, dictados, entre otros, por Jean Wahl y Paul Ricoeur.
} 
elaboran en el contexto de los cursos sobre la Pbä. $\left(1964,1968\right.$ y 1970) ${ }^{16}$ y sobre Filosofía contemporánea (1965 y 1966) que Andrés Mercado Vera dicta en la Facultad de Filosofía y Letras de la Universidad de Buenos Aires, en el marco de la cátedra de Historia de la Filosofía Moderna que estaba a su cargo y en la cual Podetti se desempeñara, primero como auxiliar docente y luego como jefa de trabajos prácticos. Dicha traducción y comentario de Phä., que en 1960 funcionan como material para los cursos en cuestión, tienen una primera publicación interna en 1978, cuando Podetti dicta su curso sobre Filosofía de la Historia centrándose en las filosofías de San Agustín y Hegel. En 2007, en oportunidad de la celebración del bicentenario de la Phä. y a veintiocho años de la muerte de la filósofa, la editorial Biblos publica el texto bilingüe de su traducción y el comentario correspondiente en un libro prologado por Jorge Mario Bergoglio y titulado Comentario a la Introducción a la Fenomenología del Espiritu.$^{17} \mathrm{La}$ edición cuenta, además, con un conjunto de textos pertenecientes al curso de 1978 y a los cuales nos referiremos en las consideraciones finales del presente trabajo.

En cuanto a la traducción, Podetti toma como fuente alemana la edición crítica de la Phä. realizada por J. Hoffmeister y publicada en 1952 en Sämtliche Werke. Neu kritische Ausgabe. Considera, además, la primera traducción castellana completa de la Phä. realizada por Wenceslao Roces con la colaboración de Ricardo Guerra, que fuera publicada precisamente en 1966 por Fondo de Cultura Económica (México). También toma en consideración la traducción parcial ya mencionada de Xavier Zubiri y las traducciones completas a otros idiomas: la francesa de J. Hyppolite, la italiana de E. De Negri y la inglesa de J.-B. Baillie. En las notas finales de su traducción, Podetti señala las diferencias con estas ediciones. El comentario, por su parte, está dividido por párrafos y tiene un carácter explicativo, aunque muy didáctico y esclarecedor. Para Podetti, la Phä. no es la historia de la razón humana en su totalidad, sino una indagación de la razón europea moderna. Pues indaga a partir de un saber absoluto ya constituido, que no es otro que la razón europea-occidental de los siglos

\footnotetext{
${ }^{16}$ El curso de 1964 significa un evento importante puesto que prácticamente no se dictaba Hegel en dicha institución desde el curso de Astrada sobre la Wissenschaft der Logik de 1955, gracias al cual Podetti tiene su primer contacto con la filosofía de Hegel.

${ }^{17}$ Esta publicación póstuma de la traducción de Podetti toma como base la interna e inédita de 1978 y dos originales mecanografiados, uno de los cuales cuenta con correcciones manuscritas.
} 
XVII y XVIII. Desde esta posición, la idea general que guía su interpretación de la "Introducción" de Phä. es que en ella se desarrolla el modo en que la filosofía crítica se refuta a sí misma y forma desde ella otra filosofía, la especulativa.

Pasemos ahora al abordaje de las tres problemáticas que guían los intereses principales de las publicaciones de la década de 1960. La primera de ellas es (i) la dialéctica de las figuras de la conciencia, tema del escrito de Astrada titulado "La dialectización de las figuras en la "Fenomenología del Espíritu", el cual forma parte de Valoración de la "Fenomenología del Espiritu". Este libro es una compilación publicada en 1964 por la editorial Devenir (Buenos Aires) y editada por Carlos Astrada, Andrés Mercado Vera, Pedro Von Haselberg, Francisco Gonzáles Ríos, Miguel Ángel Virasoro y Alfredo Llanos. El propósito general del volumen es reflejar las discusiones y posiciones dominantes de los intérpretes europeos sobre la $P b \ddot{a}$., a la que los editores argentinos califican como la obra medular del corpus hegeliano y como el libro destacado de la filosofía occidental; al tiempo que subrayan su vitalidad y actualidad para pensar los problemas más acuciantes del contexto contemporáneo y local.

El artículo de Astrada, que se concentra en las nociones de la dialéctica y las figuras de la conciencia, es el único de los seis trabajos que expone una "interpretación propia", pues no se dedica a reponer las corrientes europeas. La fuente alemana de la $P h \ddot{a}$. utilizada es la quinta edición de Sämtliche Werke, editada por Hoffmeister y publicada en 1949. Retomando críticamente las ideas que Hartmann expone en Die Philosophie des deutschen Idealismus de 1929 y en "Hegel et le problème de la dialectique du rèel" de 1931, Astrada comprende la dialéctica en la Phä. como la experiencia de la conciencia por la que ésta accede a la estructura de la realidad histórica. Las figuras de la conciencia son las formas en las que se configura la vida histórica en su propio decurso, las cuales traducen y reflejan la conciencia universal de la humanidad. Mas la originalidad de Astrada consiste en ubicar la génesis filosófica de la noción de "figura" (Gestalt) en Über die ästhetische Erziehung des Menschen in einer Reihe von Briefen de Schiller. ${ }^{18}$ El dominio de Schiller son las figuras estéticas, las cuales son siempre configuraciones espacio temporales, según interpreta Astrada, y, por lo tanto, formas determinadas, siendo lo bello la unidad reestablecida de las escisiones que el propio entendimiento genera. Para el filósofo

${ }^{18}$ La edición que utiliza es la Sämtliche Werke, Bd. IV, Tempel Verlag, Leipzig. 
argentino, Hegel traslada la idea de figura de Schiller al plano de la conciencia y a su correlato ontológico, la realidad histórica. Al igual que en el texto del '49, la figura de la conciencia destacada es la de la conciencia infeliz, aunque ahora toma un lineamiento marcadamente histórico. Aquí, sí objeta explícitamente la interpretación de Le malheur de la conscience dans la philosophie de Hegel de J. Wahl y afirma que la conciencia infeliz no se limita a ser una figura de la conciencia religiosa ni expresa necesariamente un fenómeno religioso. Constituye más bien, podríamos decir, una "figura de la conciencia histórica", pues retomando nuevamente el texto referido de Josiah Royce, el autor argentino insiste en ubicarla en el marco de la búsqueda procesual de sí mismo que el espíritu universal realiza en y a través de las escisiones de su propio devenir histórico.

La segunda (ii) cuestión de interés que se destaca en este periodo radica en los escritos juveniles de Hegel y su relación con la Phä. Esta problemática es abordada por varios trabajos de Valoración de la "Fenomenología del Espiritu". Astrada lo aborda en la última parte del texto recién mencionado, presentando las dos visiones contrapuestas que estaban en juego. Por un lado, la famosa imagen del Hegel teológico y místico que Dilthey desarrolla en Die Jugendgeschichte Hegels, y que el filósofo argentino refiere desde la edición de los Gesammelte Schriften publicados en 1929.19 Por otro lado, la imagen de un Hegel racionalista ocupado de los fenómenos sociales y económicos que Lukács expone en Derjunge Hegel, texto que Astrada trabaja desde la edición alemana publicada en 1951 por Europa Verlag (ZürichWein). Según el autor local, Lukács está en lo correcto en mostrar que las referencias del joven Hegel a los fenómenos religiosos y teológicos tienen una motivación social y política, y que el "lenguaje teológico" es más bien un modo lingüístico de época que Hegel utiliza para hablar de la situación histórica e ideológica de la Alemania del XVIII. Sin embargo, el racionalismo que Lukács atribuye a Hegel lo hace desconocer aspectos y fermentos románticos que sí son adecuadamente iluminados por Dilthey. En este sentido, el periodo juvenil de Hegel, según Astrada, no es necesariamente teológico, pero presenta elementos románticos y místicos.

En el mismo sentido lo hace Pedro von Haselberg, discípulo y colaborador alemán de Astrada, en el artículo "Bases reales de la 'Fenomenología' y la Dialéctica", donde expone sin mayores señalamientos críticos la posición que Lukács presenta en el libro ya referido; utiliza la

\footnotetext{
19 Para entonces estaba disponible la traducción castellana de este texto, realizada por Eugenio Ímaz y publicada en 1944 por Fondo de Cultura Económica.
} 
edición alemana de 1948 publicada también por Europa Verlag y de la cual presenta una reseña al año siguiente en Cuadernos de Filosofía.

El otro trabajo que se dedica a esta cuestión es "La mediación y su desenlace en la "Fenomenología" de Francisco González Ríos. El texto busca iluminar la noción de "mediación" de Hegel desde sus escritos de juventud hasta la Phä. recorriendo las tesis principales de De la médiation dans la philosophie de Hegel de Henri Niel de 1945, libro que reseña en el segundo número de Cuadernos de Filosofía en 1949. A diferencia de Astrada y Von Haselberg, González Ríos hace referencia a Hegels theologische Jugendschrifften, publicado en 1907 por Hermann Nohl, discípulo de Dilthey, en el que aparecen por primera vez los textos inéditos del joven Hegel en los que se basó el libro de su maestro. ${ }^{20} \mathrm{El}$ artículo despliega la tesis de que la concepción hegeliana de la mediación, inescindible de la de "superación" (Aufhebung), se desarrolla en el joven Hegel a partir de la direccionalidad religiosa del espíritu, desde la que busca hallar los fundamentos racionales de los dogmas religiosos. Tal direccionalidad, la cual cobra en dicho periodo un aspecto histórico que se manifiesta en el interés hegeliano por la religión total y en nociones como las de "espíritu del pueblo" (Volksgeist) y "espíritu del mundo" (Weltgeist), halla su maduración metafísica en la Phä. en cuanto irrupción en el plano de lo absoluto. La mediación se consolida aquí como el movimiento racional -reflexivo y negativo- de lo absoluto, constituyendo a la $P h \ddot{a}$. como un esbozo del sistema especulativo total.

El cuarto y último trabajo sobre el tema es "El joven Hegel y la 'Fenomenología del Espíritu"' de Alfredo Llanos, quien estuvo a cargo de la primera traducción argentina completa de la $P h \ddot{a}$., publicada por editorial Rescate (Buenos Aires) en 1991. El texto repone críticamente Le jeune Hegel et la visión morale du monde de Adrien Peperzak, que aparece en 1960. El propósito de ese libro, según reconstruye Llanos, es evitar las lecturas excesivamente teológicas de los escritos juveniles de Hegel, como también las excesivamente políticas como la de Lukács. Su peculiaridad radica en poner en el centro del debate la discusión de Hegel con la filosofía práctica de Kant y el progresivo rechazo de su abstracción y de sus dualismos

\footnotetext{
${ }^{20}$ A diferencia de las otras contribuciones, la de González Ríos presenta la lista de las ediciones alemanas de la obra completa de Hegel que estaban disponibles. Véase: González Ríos, Francisco, "La mediación y su desenlace en la "Fenomenología"', en Carlos, Astrada, Andrés, Mercado Vera, et. al. (Eds.), Valoración de la "Fenomenología del Espíritu", Buenos Aires, Editorial Devenir, 1964, pp. 104-106.
} 
concomitantes desde una filosofía de la reconciliación que busca, desde el plano religioso, desvelar la realidad social y política junto a la libertad humana. Desde esa perspectiva, Hegel se debate entre dos concepciones del mundo entre 1790 y 1800: una totalitaria, reflejada en su adhesión a la concepción del pueblo grande y libre de inspiración griega, y una moralista, centrada en la noción de libertad de la Revolución francesa. Estos dos ideales alcanzan su reconciliación reflexiva en Systemfragment de 1800, el cual constituye una antesala fundamental de la $P h a ̈$., en la que el movimiento de unificación adquiere su madurez y se refleja en un humanismo místico. Ahora bien, según Llanos, el problema fundamental del trabajo de Peperzak es que comprende la reconciliación desde un punto de vista religioso, lo que hace que su interpretación general del periodo juvenil de Hegel quede apegada al lineamiento teológico. Según el autor argentino, quien sugiere leer dicho periodo desde los textos de madurez de Hegel, el lineamiento teológico se anularía con la superación filosófica de la religión del periodo de madurez. En contraposición, el autor argentino acentúa el carácter negativo y dialéctico del pensamiento de Hegel, así como su impulso revolucionario. Se trata, para él, de un humanismo integral que logra conciliar dialécticamente los ideales antes mencionados, pero desde el posicionamiento ateo subrayado por Kojève.

El tercer (iii) foco de interés de este periodo son las interpretaciones francesas, abordadas por las dos restantes colaboraciones del volumen que nos ocupa. Una de ellas es "Una nueva interpretación de la 'Fenomenología"' de Virasoro, que repone algunas relaciones que Jean Hyppolite establece entre Hegel y la filosofía existencial en Génese et structure de la Phénomenologie de l'Esprit de Hegel -obra que se publica en 1946-. El artículo enfatiza que una de las mayores originalidades de Hegel es haber mostrado la identidad del saber de sí y el saber de otro, desde la cual se comprende la desigualdad de la conciencia con su objeto y consigo misma como un momento del movimiento configurador del saber. Ello se pone de manifiesto en la famosa lucha de las conciencias y la exigencia de reconocimiento recíproco expuestas en el capítulo IV, temática que Heidegger retoma con la noción de "ser-con" y Sartre con el fenómeno de la mirada. Pero también, en términos más generales, aquello se muestra en la totalidad del camino de la conciencia natural hacia el saber absoluto, en el que ella experimenta la desesperación de la desigualdad que, desde la filosofía de la existencia, se caracteriza como angustia existencial. Esta filosofía, contrapuesta al posicionamiento especulativo, privilegia el aspecto 
negativo, es decir, la desigualdad. Esta posición queda representada paradigmáticamente por el desgarramiento originario de la ontología sartreana, que hace imposible la síntesis del ser-en-sí y del ser-para-sí, dejando a la conciencia en el plano de la permanente desventura. El otro punto que el trabajo destaca es el posicionamiento de Hegel contra la crítica de Kierkegaard, según la cual el individuo universal hegeliano niega y anula la conciencia individual. Como señala Hyppolite, la historia de formación del individuo que expone la $P h \ddot{a}$. debe entenderse más bien como el recorrido por el que la conciencia individual alcanza el saber de sí en la inmanencia de la historia espiritual de la humanidad; universalidad en la que aquella no es negada, sino en la que se realiza plenamente como individualidad. No hay, pues, conciencia individual sin conciencia universal, por lo que la crítica de Kierkegaard quedaría refutada.

La segunda contribución es "Antropogonía, dialéctica y muerte en la 'Fenomenología del Espíritu”' de Andrés Mercado Vera, quien repone de manera expositiva la interpretación antropológica de la $P h \ddot{a}$., desarrollada por Kojève en Introduction á la lecture de Hegel-texto que se publica en $1947 \mathrm{y}$ contiene parte de los cursos que dicta en la década de 1930 en la École des Hautes Études-. El artículo de Vera se detiene en el famoso análisis kojèveano del capítulo IV, prestando especial atención a la concepción del deseo de reconocimiento como deseo antropógeno, a la dialéctica de la dominación y la servidumbre como lógica estructurante de la historia universal, y a la liberación del esclavo y el concomitante nacimiento de las ideologías, es decir, de los relatos que intentan conciliar la mera idea de libertad con el hecho efectivo de la servidumbre. En segundo lugar, Vera se ocupa del tránsito del Estado pagano al cristianismo, el cual constituye un reflejo de las relaciones de dominación y servidumbre del primero, pero desde el plano de la trascendencia divina. La superación de la dominación y la servidumbre, para la que es menester la superación del cristianismo, se establece en el Estado absoluto de Napoleón, el cual marca el fin de la historia en tanto realización de la libertad y de las individualidades reconocidas recíprocamente. El ateísmo de la filosofía especulativa de Hegel permite, a la vez, una comprensión de la muerte como negación dialéctica y no como paso a un más allá trascendente ni como mero acontecer natural. Finalmente, Vera insiste en que la dialéctica fenomenológica no constituye un método, sino más bien la actitud contemplativa por la que el ser metafísico y ontológico aparece al hombre, punto en el que se diferencia del texto de Virasoro recién reseñado, que 
vuelve sobre la idea según la cual el método de la Phä. consiste en el movimiento constructivo de la razón.

El cuarto (iv) y último foco de interés de esta etapa es la relación entre Hegel y Marx. Héctor Raurich se ocupa del ello en Notas para la actualidad de Hegel y Marx, que se publica en 1968 en el marco de la primera etapa de la dictadura militar de Juan Cargos Onganía (1966-1970). Se trata de una publicación póstuma que reúne las notas redactadas por el autor entre 1961 y 1963 , esto es, los últimos tres años de su vida. ${ }^{21}$ El libro no se centra exclusivamente en la $P b \ddot{a}$., sobre la cual hay referencias directas e indirectas. Reflexiona sobre distintos temas del pensamiento de Hegel y remite a varias de sus obras, aunque de manera muy general. Lo mismo sucede con los Manuscritos de 1844 y Das Kapital de Marx. En ningún caso se consignan las ediciones utilizadas. Las Notas de Raurich presentan una lectura históricomarxista de Hegel. Aunque las relaciones que establece con Marx son bastante generales, su propuesta es concisa: “"marxistizar' a Hegel y 'hegelianizar' a Marx". 22 Raurich rechaza la contraposición entre Hegel, filósofo del pensar, y Marx, filósofo de la praxis, e insiste en su compatibilidad. Entiende la metafísica hegeliana como una metafísica de la historia, cuyo protagonista es el espíritu concreto, es decir, el hombre social y comunitario, no el espíritu universal abstracto, como sugería Astrada en Marx y Hegel. Tanto Hegel como Marx, según reza la tesis principal del trabajo respecto del tema que nos convoca, son pensadores de su tiempo: el tiempo de la revolución, el cual es también el de la contemporaneidad. Ello implica que, como señala Hegel en la Phä., se asiste al fin de una época en la que la humanidad ha adquirido el saber absoluto de sí misma, dando

${ }^{21}$ En 1959, dos años antes de comenzar estas notas, Raurich obtiene el cargo de Profesor Adjunto de Filosofía del Derecho en la Facultad de Derecho de la Universidad Nacional de La Plata, momento y lugar en el que dicta el curso "La filosofía de Marx en su relación con la Filosofía de Hegel". Durante la década de 1950 dicta varios cursos sobre Hegel en diversas instituciones, que fueron reunidos y publicados por sus discípulos en 1976 en Hegely la lógica de la pasión. Notas contiene, entre otras cosas, un apéndice dedicado a Croce, donde le objeta dejar a la Phä. en un segundo plano y desvirtuar las ideas hegelianas. También hace una breve mención al debate sobre los escritos juveniles de Hegel, oponiéndose a la lectura teológica.

${ }^{22}$ Raurich, Héctor, Notas para la actualidad de Hegel y Marx, Santa Fe - Buenos Aires, Editorial Marymar, 1968, p. 34. 
paso, desde la Revolución francesa, al inicio de una reconfiguración histórica en la que se debate el tiempo presente.

La otra obra que se ocupa de esta cuestión es Dialéctica e Historia. Hegel -Marx de Astrada, publicada en 1969 por Juárez Editor (Buenos Aires). El libro vuelve sobre algunos puntos de Marx y Hegel de 1958, para retomar su lineamiento interpretativo histórico-marxista. En este caso, aborda la concepción de la historia de ambos filósofos desde las ideas axiales de razón y libertad. Según el autor argentino, la Phä. constituye la primera Filosofía de la historia de Hegel. ${ }^{23}$ Se trata de la historia universal del espíritu en la que el individuo se forma culturalmente, es decir, conquista el saber de su propia historia, la historia conceptualizada o saber absoluto. Este término no constituye, como sugiere Kojève, el fin del tiempo histórico. Apoyándose en Hegel. Die grebrochene Mitte de Jan van der Meulen (1958), lo comprende como el punto de discontinuidad del decurso histórico, el cierre de sus figuras reiterables que abre paso a la realización de la libertad, telos que estructura el desarrollo de la historia universal y que, a través de la Revolución francesa, hallaría su cauce de realización en la sociedad burguesa. ${ }^{24}$ Sin embargo, Astrada repite la crítica al idealismo hegeliano que había presentado en el texto de 1958 a través de los Manuscritos de 1844 de Marx ${ }^{25}$, lo que lo distancia de la postura más conciliadora de Raurich. En primer lugar, Hegel hipostasia las estructuras lógicas ideales y las transfiriere al terreno de la historia como formas universales del pensar, olvidando los factores materiales, iluminados por el realismo marxista y su énfasis en la praxis concreta de la historia humana. En segundo lugar, retira la objeción a la concepción hegeliana de la libertad, a la que vuelve a caracterizar como abstracta y meramente cognitiva, contraponiéndole la visión marxista. Pero ahora refiere explícitamente al Posfacio de la segunda edición de Das Kapital para enfatizar la idea de que la verdadera liberación del hombre no se realiza en la sociedad burguesa, sino en una sociedad sin clases producto de la transformación revolucionaria de los modos capitalistas de producción

\footnotetext{
${ }^{23}$ La fuente alemana de la Phä. utilizada es la misma que en "La dialectización de las figuras en la 'Fenomenología del Espíritu"'. Cf. supra.

${ }^{24}$ Cabe señalar que, en este libro, Astrada refiere también a otras obras de Hegel, sobre todo a Vorlesungen über die Philosophie der Geschichte, Reclam Verlag, 1961 y a Grundlinien der Philosophie des Rechts, Sämtliche Werke, Bd. 7, Hg. von H. Glockner, Stuttgart, Felix Meiner, 1927.

${ }^{25}$ La edición alemana de los Manuscritos es la misma que en Marx y Hegel. Cf. supra.
} 
llevada a cabo por el proletariado. ${ }^{26}$ Finalmente, tras las fuertes críticas realizadas, Astrada recurre a Hegel und die französische Revolution de Ritter, en su edición de 1965 y califica a Hegel como "el primer filósofo moderno de la revolución continuada e ininterrumpida". ${ }^{27}$ En efecto, fue él quien vio el mundo moderno como un viejo mundo, en el que la consolidación de sus estructuras son también su propio principio de ruina. Por esto mismo, fue Hegel quien, sin caer en la profecía filosófica, supo visualizar que el tiempo venidero, nuestro presente, es el destino del cumplimiento de la modernidad y, por lo tanto, el tiempo de transición hacia un nuevo mundo.

\section{Consideraciones finales}

La década de 1960, etapa políticamente convulsionada del país, constituye un periodo muy fructífero de los estudios argentinos dedicados a la Phä., especialmente entre 1964 y 1969. En general, no tienen el registro de lo que hoy se encamararía dentro de la Hegelforschung especializada y academicista, la cual se consolida en Argentina a partir de la década de 1980, pues presentan un abordaje más bien apropiativo y no exegético de la obra de Hegel. El lineamiento interpretativo dominante es el histórico en un doble sentido. En primer lugar, la historia conforma una temática central y un aspecto fundamental desde el que se establecen las lecturas de la Phä. Esto se refleja en el carácter histórico que Astrada le otorga a la figura de la conciencia infeliz en su artículo de 1964, que desarrolla lo que hemos delimitado como el primer foco temático de interés de este periodo, la dialéctica de las figuras de la conciencia. Se pone de relieve también y de modo paradigmático en las publicaciones que abordan la cuarta problemática más acuciante de esta etapa, la relación entre Hegel y Marx. Las Notas de Raurich de 1961-1963 (publicadas en 1968) y el libro de Astrada de 1969 exponen lecturas marxistas de Hegel en las que la historia se erige como un eje fundamental de la indagación. En segundo lugar, la impronta histórica toma la forma de "relevamiento histórico". Esto sucede en las contribuciones que conforman la compilación de 1964, Valoración de la "Fenomenología del Espiritu", las cuales reponen las interpretaciones europeas más relevantes sobre el segundo y tercer foco de interés de los

${ }^{26}$ La fuente alemana de Das Kapital es la publicada en 1967 por Europäische Verlagsanstalt.

${ }^{27}$ Astrada, Carlos, Dialéctica e historia. Hegel-Marx. Buenos Aires, Juárez Editor, 1969, p. 144. 
años 60: los escritos juveniles de Hegel, su relación con la Phä. y las interpretaciones francesas.

La producción de esta etapa muestra un gran conocimiento por parte de los intelectuales argentinos de las problemáticas más acuciantes en torno a los cuales giraban por entonces las investigaciones globales sobre la Phä. Ello se exhibe en las temáticas abordadas, en los interlocutores y autores aludidos, cuyas obras son referidas mayormente en su idioma original. Las fuentes de la $P h a ̈$. utilizadas son también las ediciones alemanas actualizadas.

La vitalidad y la potencia que presenta la $P h \ddot{a}$. para pensar problemáticas contemporáneas es uno de los puntos más valorados y enfatizados por las publicaciones argentinas. Ramiro Podetti afirma que la cuestión de base del Primer Congreso Nacional de Filosofía de 1949 radicó en la búsqueda de una filosofía regional, es decir, soluciones propias a los avatares políticos y sociales del país o de Latinoamérica. ${ }^{28}$ Consideramos que esto vale también para los textos de la década de 1960, pues manifiestan, en algunos casos explícitamente y en otros implícitamente, un espíritu de reapropiación local del pensamiento de Hegel. En Dialéctica e Historia, Astrada menciona problemáticas puntuales que funcionan de telón de fondo de su estudio comparativo entre Hegel y Marx. Entre ellas se destacan no sólo el destino del socialismo, sino también la rebelión mundial del poder estudiantil, las migraciones externas e internas en el continente americano y los conflictos raciales. Temas, sin duda, de profunda actualidad, que incluso hoy marcan parte de la agenda política del mundo.

Pero una peculiaridad de esta apropiación local es que no constituye un localismo cerrado en sí mismo. Las investigaciones hegelianas argentinas de los años 60 constituyen un pensamiento situado que, a la vez, se inserta en los debates internacionales. Los textos de Amalia Podetti, que acompañan la publicación de su traducción de la "Introducción" a la Phä. y el comentario correspondiente, teorizan bien esta cuestión. Agrupados bajo el título "La Fenomenología del espiritu y la Historia de Occidente", explican "la actualidad de la Fenomenología del espiritu en función de la tensión hacia el universalismo y de nuestra situación como argentinos y americanos". ${ }^{29}$ Aquellos textos, como ya anticipamos, pertenecen a una clase sobre el

${ }_{28}$ Podetti, Ramiro, “Advertencia preliminar", en Amalia, Podetti, Comentario a la Introducción a la Fenomenología del Espíritu, Buenos Aires, Biblos, 2007, p. 30.

${ }^{29}$ Aimetta de Colotti, Ana María, "Aproximación a la Fenomenología del Espíritu”, en Amalia, Podetti, Comentario a la Introducción a la Fenomenología del Espíritu, Buenos Aires, Biblos, 2007, p. 35. 
sentido y la ubicación de la Phä. que Podetti brinda en el marco de su curso de Filosofía de la Historia en 1978. ${ }^{30}$ Sin embargo, se publican junto a la traducción de 1966 y al comentario de 1968 porque son representativos de la aproximación de la autora al pensamiento de Hegel.

Podetti sostiene que una de las tesis fundamentales de la Phä. para pensar la historia es la famosa idea, retomada por varios autores, según la cual se asiste al fin de una época, la del mundo moderno y la cultura occidental, y a la gestación de una nueva configuración espiritual. Desde el punto de vista hegeliano, la historia de Occidente constituye un proceso de universalización que está llegando al cumplimiento de su realización en la contemporaneidad, tal como lo hace patente la globalización. Ahora bien, en el proceso de universalización, la irrupción de América ha jugado un papel central, pues con ella comienza la historia universal. La concepción de Hegel sobre América, que se expone en sus Lecciones, no es del todo positiva. La ubica más bien del lado de la naturaleza, en la que aún el espíritu no ha producido nada. Esta visión negativa se refleja en la perspectiva hegemónica europea que ha imperado por muchos años y que tiene una dimensión doble: América, como el mundo desconocido y, luego, como una extensión de Europa, perspectiva a la cual Astrada califica de "provincialismo miope". ${ }^{31}$ Sin embargo, Podetti recuerda que, para Hegel, América es también quizá la tierra del porvenir. Constituye simultáneamente un término histórico y geográfico porque es el último lugar a donde llega el hombre europeo en el proceso histórico de ocupación terrestre. El cumplimiento de la universalización halla aquí su sitio. Pero como término o límite culminante, América es un punto de vista (Standpunkt) privilegiado para pensar la universalización y, por lo tanto, es también comienzo de la reconfiguración de un nuevo mundo. Así, es posible comprender la recepción argentina de Hegel y, específicamente, la de la $P h a ̈$., como parte de un proceso histórico en el que la conciencia americana se hace partícipe de la historia global y toma un lugar activo, configurador en el movimiento de la universalización contemporánea.

\footnotetext{
${ }^{30}$ Los escritos en cuestión son tres: "El proceso de universalización”, "La aparición de américa en la Historia" y "El marco histórico de la Fenomenología del Espiritu". El segundo de ellos tuvo una primera publicación póstuma a través del Centro de Investigaciones Culturales del que formaba parte Aimetta de Colotti.

31 Astrada, Carlos, Dialéctica e historia; Hegel - Marx. Buenos Aires, Juárez Editor, 1969, p. 101.
} 


\section{Referencias bibliográficas}

- Aimetta de Colotti, Ana María, "Aproximación a la Fenomenología del Espiritu", en Amalia, Podetti, Comentario a la Introducción a la Fenomenología del Espíritu, Buenos Aires, Biblos, 2007, pp. 35-46.

- Alberini, Coriolano, "Prólogo", en Luis Farré, Cincuenta años de filosofía en Argentina, Buenos Aires, Peuser, 1958, pp. 7-19.

- Astrada, Carlos, Dialéctica e bistoria; Hegel - Marx. Buenos Aires, Juárez editor, 1969.

- Astrada, Carlos, Mercado Vera, Andrés, et. al. (Eds.), Valoración de la "Fenomenologia del Espiritu”, Buenos Aires, Editorial Devenir, 1964.

- Astrada, Carlos, "La dialectización de las figuras en la 'Fenomenología del Espíritu", en Carlos, Astrada, Andrés, Mercado Vera, et. al. (Eds.), Valoración de la "Fenomenología del Espiritu", Buenos Aires, Editorial Devenir, 1964, pp. 1-21.

- Astrada, Carlos, Marx y Hegel. Trabajo y alienación en la "Fenomenología" y en los "Manuscritos", Buenos Aires, Siglo Veinte, 1958.

- Astrada, Carlos, El marxismo y las escatologías, Buenos Aires, Procyon, 1957.

- Astrada, Carlos, Hegely la dialéctica, Buenos Aires, Kairós, 1956.

- Astrada, Carlos, "Génesis dialéctica de la conciencia infeliz", Serie Textos Filosóficos. Filosofía Moderna, 3 (1949), pp. 5-10.

- Croce, Benedetto, Ciò che è vivo e ciò che è morto della filosofia di Hegel, en Saggio sullo Hegel, Bari, Laterza \& Figli, 1932.

- Dilthey, Wilhelm, Gesammlete Sschriften, Bd. IV, Die Jugendgeschichte Hegels, Berlín, 1929 (Hegel y el idealismo, Trad de Eugenio Ímaz, México, Fondo de Cultura Económica, 1944).

- Gentile, Giovanni, La Riforma della Dialettica Hegeliana, 2da. ed., Messina, Principato, 1923.

- González Ríos, Francisco, "La mediación y su desenlace en la 'Fenomenología"', en Carlos, Astrada, Andrés, Mercado Vera, et. al. (Eds.), Valoración de la "Fenomenología del Espíritu", Buenos Aires, Editorial Devenir, 1964, pp. 68-114. 
- González Ríos, Francisco, "De la médiation dans la philosophie de Hegel de Henry Niel (1945)”, Cuadernos de Filosofía, 2 (1949).

- Hartmann, Nicolai, Die Philosophie des deutschen Idealismus, Berlín, Gruyter, 1929.

- Hartmann, Nicolai, "Hegel et le problème de la dialectique du rèel”, Etudes sur Hegel. Revue de métaphysique et de morale, 38-3 (1931), pp. 285-316.

- Hegel, Georg Wilhelm Friedrich, Fenomenología del Espiritu, Trad. A. Llanos, Buenos Aires, Rescate, 1991.

- Hegel, Georg Wilhelm Friedrich, Fenomenología del Espíritu, Trad. W. Roces con colaboración de R. Guerra, México, Fondo de Cultura Económica, 1966.

- Hegel, Georg Wilhelm Friedrich, Vorlesungen über die Philosophie der Gescbichte, Reclam Verlag, 1961.

- Hegel, Georg Wilhelm Friedrich, Sämtliche Werke. Neu kritische Ausagbe, J. Hoffmeister (hrsg.), Hamburg, Felix Meiner, 1952.

- Hegel, Georg Wilhelm Friedrich, "La conciencia infeliz", Serie Textos Filosóficos. Filosofía Moderna, 3 (1949), Traductor desconocido, Facultad de Filosofía y Letras, Instituto de Filosofía de la Universidad de Buenos Aires, Buenos Aires, pp. 11-33.

- Hegel, Georg Wilhelm Friedrich, La Phénoménologie de l'Esprit, Trad. J. Hyppolite, 2 t., París, Aubier, 1939 (Segunda edición: París, Éditions Montaigne, 1941).

- Hegel, Georg Wilhelm Friedrich, Fenomenología del Espíritu. Prólogo. Introducción. Saber absoluto, Trad. de Xavier Zubiri, (1935), Revista de Occidente; Madrid, pp. IX-XVI.

- Hegel, Georg Wilhelm Friedrich, Fenomenologia dello Spirito, Trad. Enrico de Negri, 2 Vol., Florencia, La Nuova Italia Editrice, 1933 (Segunda edición revisada, 1949).

- Hegel, Georg Wilhelm Friedrich, Sämtliche Werke. Jubiläumsausgabe in zwañig Bänden, Bd. II, Neu hg. Von H. Glockner, Stuttgart, 1932.

- Hegel, Georg Wilhelm Friedrich, Grundlinien der Philosophie des Rechts, Sämtliche Werke, Jubiläumsausgabe im zwanzig Bänden, Bd. 7, Neu Hg. von H. Glockner, Stuttgart, Felix Meiner, 1927. 
- Hegel, Georg Wilhelm Friedrich, The Phenomenology of Mind, Trad. J.-B. Baillie, Londres Library of Philosophy, 1910 (Edición revisada, 1931).

- Hegel, Georg Wilhelm Friedrich, Sämtliche Werke, Bd. II, Hg. von G. Lasson, später J. Hoffmeister, Leipzig-Hamburg, Felix Meiner, 1907 (Otras ediciones referidas: 1921, 1937, 1949).

- Hegel, Georg Wilhelm Friedrich, Filosofia del espiritu, Trad. de Eduardo Barrioberro y Herrán, Madrid, Daniel Jorro, 1907 (Reediciones: Ed. Anaconda, Buenos Aires, 1941; Ed. Claridad, Buenos Aires, 1969 y 2006).

- Hegel, Georg Wilhelm Friedrich, Philosophie de l'esprit, Trad. de Augusto Véra, París, Germer Baillière, 1869 (Reedición, 1897).

- Hegel, Georg Wilhelm Friedrich, Werke. Vollständige Ausgabe durch einen Verein von Freunden des Verewigten. Bd. II, Berlín, Duncker und Humblot, 1832 (Segunda edición 1841).

- Hegel, Georg Wilhelm Friedrich, System der Wissenschaft. Erster Theil. Phänomenologie des Geistes, Bamberg und Würzburg, bey J. A. Goebhardt, 1807

- Hegel, Georg Wilhelm Friedrich, Fenomenología del Espiritu, Trad. A. Gómez Ramos, Madrid, Abada, 2010. Edición bilingüe que sigue la edición canónica realizada por W. Bonsiepen y R. Heede, Gesammelte Werke, Band 9, Hamburg, 1980.

- Hyppolite, Jean, Génese et structure de la Phénomenologie de l'Esprit de Hegel, París, Aubier-Montaigne, 1946.

- Kojève, Alexandre, Introduction á la lecture de Hegel, París, Gallimard, 1947.

- Llanos, Alfredo, "El joven Hegel y la Fenomenología del Espíritu", en Carlos, Astrada, Andrés, Mercado Vera, et. al. (Eds.), Valoración de la "Fenomenología del Espiritu", Buenos Aires, Editorial Devenir, 1964, pp. 130150.

- Lukács, Georg, Der junge Hegel-Über die Beriehungen von Dialektik und Ökonomie, Zürich-Wein, Europa Verlag, 1948 (Reedición, 1951).

- Marx, Karl, Das Kapital, Bd. I, Nachwort zur zweiten Auflage, Europäische Verlagsanstalt, 1967. 
- Marx, Karl, National Oekonomie und Philosophie, Verlag Kiepenheuer, Köln, 1950. Texto que reproduce el de la Marx-Engels-Ausgabe. Instituto MarxEngels-Lenin, Moscú. Bd. 3, 1932.

- Mercado Vera, Andrés, "Antropogonía, dialéctica y muerte en la Fenomenología del Espíritu”, en Carlos, Astrada, Andrés, Mercado Vera, et. al. (Eds.), Valoración de la "Fenomenología del Espiritu", Buenos Aires, Editorial Devenir, 1964, pp. 22-54.

- Meulen, Jan van der, Hegel. Die grebrochene Mitte, Hamburg, Felix Meiner, 1958.

- Niel, Henri, De la médiation dans la philosophie de Hegel, Editions AubierMontaigne, París, 1945.

- Nohl, Hermann, Hegels theologische Jugendschriften, Tubingen, 1907.

- Peperzark, Adrien, Le jeune Hegel et la visión morale du monde, La Haye, Martinus Nijhoff, 1960.

- Podetti, Amalia, Comentario a la Introducción a la Fenomenología del Espiritu, Buenos Aires, Biblos, 2007.

- Podetti, Ramiro, “Advertencia preliminar", en Amalia, Podetti, Comentario a la Introducción a la Fenomenología del Espíritu, Buenos Aires, Biblos, 2007, pp. 15-34.

- Ramaglia, Dante, "Apropiación crítica de Hegel en la filosofía argentina contemporánea”, Cuyo. Anuario de Filosofía Argentina y Americana, 24 (2007), pp. 121-137.

- Raurich, Héctor, Hegel y la lógica de la pasión, Santa Fe - Buenos Aires, Editorial Marymar, 1976.

- Raurich, Héctor, Notas para la actualidad de Hegely Marx, Santa Fe - Buenos Aires, Editorial Marymar, 1968.

- Ritter, Joachim, Hegel und die französische Revolution, Frankfurt am Main, Shurkamp, 1965.

- Royce, Josiah, Lectures on Modern Idealism, Ed. J. Loewenberg, New Haven, Yale University Press, 1919 (Reediciones, 1923, 1945. Traducción castellana: El idealismo moderno, Trad. Vicente Quintero, Buenos Aires, Imán, 1945). 
- Sepich, Juan Ramón, Propedéutica filosófica. Prefacio al "Sistema de la ciencia" de Hegel, Buenos, Aires, Itinerarium, 1972.

- Schiller, Friedrich, Sämtliche Werke, Bd. IV, Über die ästhetische Erziehung des Menschen in einer Reibe von Briefen, Leipzig, Tempel Verlag.

- Virasoro, Miguel Ángel, "Una nueva interpretación de la 'Fenomenología"', en Carlos, Astrada, Andrés, Mercado Vera, et. al. (Eds.), Valoración de la "Fenomenología del Espíritu", Buenos Aires, Editorial Devenir, 1964, pp. 116-129.

- Virasoro, Miguel Ángel, "Guido de Ruggiero”, en Actas del Primer Congreso Nacional de Filosofía, Tomo I, Universidad Nacional de Cuyo, Mendoza, 1950, pp. 607-610.

- Von Haselberg, Pedro, "Bases reales de la 'Fenomenología' y la Dialéctica", en Carlos, Astrada, Andrés, Mercado Vera, et. al. (Eds.), Valoración de la "Fenomenología del Espíritu", Buenos Aires, Editorial Devenir, 1964, pp. 55-67.

- Von Haselberg, Pedro, "Der junge Hegel-Über die Beziehungen von Dialektik und Ökonomie”, Cuadernos de Filosofía, 2 (1949).

- Wahl, Jean, Le Malheur de la Conscience dans la Philosophie de Hegel, París, Presses Universitaires de France, 1929.

Recibido: 04/2021; aceptado: 07/2021 